\title{
Calidad de vida y salud bucal: Perspectiva de adultos mayores atendidos en la red hospitalaria pública de Medellín, Colombia*
}

\section{Quality of Life and Oral Health: Perspectives of the Elderly Population Assisted by the Public Hospital Network of Medellín, Colombia}

\section{Qualidade de vida e saúde bucal: Perspectiva de idosos atendidos na rede hospitalar pública de Medellín, Colômbia}

Fecha de recepción: 02-06-15 Fecha de aceptación: 18-08-15 Disponible en línea: 01-11-15 doi: 10.11144/Javeriana.rgyps14-29.cbsv

Cómo citar este artículo:

Alzate-Urrea S, Agudelo-Suárez AA, López-Vergel F, López-Orozco C, Espinosa-Herrera E, PosadaLópez A. Calidad de vida y salud bucal: Perspectiva de adultos mayores atendidos en la red hospitalaria pública Rev. Gerenc. Polít. Salud. 2015; 14(29): 83-96. http://dx.doi.org/10.11144/Javeriana.rgyps14-29.cbsv

\author{
Santiago Alzate-Urrea** \\ Andrés A. Agudelo-Suárez*** \\ Felipe López-Vergel ${ }^{* *}$ \\ Carolina López-Orozco** \\ Érica Espinosa-Herrera** \\ Adriana Posada-López $* * * *$ \\ Edwin J. Meneses-Gómez*****
}

Artículo derivado de la investigación titulada Determinantes de la calidad de vida y su impacto en la salud bucal de la población adulta mayor que consulta a la E.S.E Metrosalud: Una perspectiva de género. Inicio: 27-12-2011, finalización: 27-082014, financiación: E.S.E Metrosalud, Medellín (código: C02-E11-L3-01) y facultades de Odontología de las instituciones participantes. Autor principal y de correspondencia: Santiago Alzate Urrea, Facultad de Odontología de la Universidad de Antioquia, Calle 70 No. 52- 21, Medellín, Colombia. Correo electrónico: santiagoalzatte@gmail.com

* Odontólogos de la Universidad de Antioquia, Medellín, Colombia. Correos electrónicos: santiagoalzatte@gmail.com, jfelipe23@hotmail.com, carolop3z@hotmail.com y erika231291@hotmail.com

*** Odontólogo, Ph.D. en Salud Pública, profesor asociado de la Facultad de Odontología de la Universidad de Antioquia. Correo electrónico: oleduga@gmail.com

***** Odontóloga y licenciada en Biología y Química, magíster en Epidemiología, profesora de la Facultad de Odontología de la Universidad de Antioquia, profesora de la Fundación Universitaria Autónoma de las Américas. Correo electrónico: adriposalo@gmail.com

******* Odontólogo, Ph.D en Ciencias Odontológicas, profesor de la Facultad de Odontología de la Fundación Universitaria Autónoma de las Américas, sedes Medellín y Pereira. Correo electrónico: edjamego@yahoo.com 


\section{Resumen}

Objetivo: analizar la percepción de la población adulta mayor sobre su calidad de vida, relacionándola con su estado de salud general y bucal, desde el discurso de sus actores primarios. Materiales y métodos: estudio cualitativo etnográfico, se realizaron 13 grupos focales (GF) en población adulta $\geq 65$ años, asistentes a la E.S.E. Metrosalud (Medellín). Se realizó transcripción textual de los GF y análisis de contenido según categorías, identificando puntos de acuerdo, disenso y tendencias en la información. Resultados: se analizaron cinco categorías: salud autopercibida y factores que influyen en el estado de salud; calidad de vida, calidad de vida con la salud y calidad de vida relacionada con la salud bucal; apoyo familiar y social; envejecimiento saludable; y acceso a los servicios de salud. Conclusión: la autopercepción sobre la calidad de vida es un aspecto fundamental para evaluar los determinantes de la salud en la población adulta mayor.

Palabras clave: adulto mayor; calidad de vida; salud de la persona mayor; salud bucal; poblaciones vulnerables; servicios de salud para ancianos (Fuente: DeCS- Bireme)

\section{Abstract}

Objective: To analyze the perception of older adults on their quality of life, relating it to your general and oral health, from the speech of its primary actors. Materials and methods: qualitative ethnographic study, 13 focus groups ( $\mathrm{FG}$ ) were performed in adults $\geq 65$ years attending the ESE Metrosalud (Medellin). GF verbatim transcript and content analysis was performed according to categories, identifying points of agreement, dissent and trends in the data. Results: were found and analyzed five categories: self-perceived health and factors affecting health status; quality of life, quality of life, health and quality of life related to oral health; family and social support; healthy aging; and access to health services. Conclusion: The perception of quality of life is crucial for assessing the determinants of general and oral health in the older population aspect.

Keywords: aged; quality of life; health of the elderly; oral health; vulnerable populations; health services for old people

\section{Resumo}

Objetivo: analisar a percepção da população de idosos sobre sua qualidade de vida, relacionando-a com o seu estado de saúde geral e bucal, desde o discurso dos seus atores primários. Materiais e métodos: estudo qualitativo etnográfico, realizaram-se 13 grupos focais (GF) com população adulta $\geq 65$ anos, assistentes à E.S.E. Metrosalud (Medellín). Transcrição textual dos GF e análise de conteúdo de acordo com categorias foi feito, identificando pontos de concordância, discordância e tendências dos dados. Resultados: analisaram-se cinco categorias: saúde autopercebida e fatores que influenciam o estado de saúde; qualidade de vida, qualidade de vida com saúde e qualidade de vida relacionada com a saúde bucal; apoio familiar e social; envelhecimento saudável; e acesso a serviços de saúde. Conclusão: a autopercepção sobre a qualidade de vida é aspecto fundamental para avaliar as determinantes da saúde na população idosa.

Palavras-chave: idoso, qualidade de vida, saúde do idoso, saúde bucal, populações vulneráveis, serviços de saúde para idosos (Fonte: DeCS- Bireme) 


\section{Introducción}

La población latinoamericana viene experimentando un cambio en sus indicadores demográficos durante los últimos años y uno de ellos guarda relación con el envejecimiento de la población, traducido en una mayor esperanza de vida y en la proporción de personas consideradas como adultos mayores (1). En Colombia, según indicadores de instituciones internacionales, la esperanza de vida ha aumentado de 71 a 74 años en el periodo 2000-2012 (de 75 a 78 años en el caso de las mujeres y de 67 a 70 años en los hombres) (2). En porcentaje, aproximadamente el $7 \%$ de la población colombiana es mayor de 65 años (3). Paralelamente a este fenómeno, también se han incrementado los niveles de pobreza y de vulnerabilidad social, instaurándose así a la población adulta mayor como endeble y excluida, características que afectan su calidad de vida (4-6).

Un primer elemento, la vulnerabilidad social, hace alusión al carácter de las estructuras e instituciones económico-sociales y al impacto que tienen en comunidades, familias y personas en distintas dimensiones de la vida social (7). El contexto biológico, cultural, social y político de algunos subgrupos de la población adulta mayor, los pone en situación de vulnerabilidad, ya que se puede presentar un descenso del flujo de sus relaciones sociales, algunas alteraciones biológicas en su organismo y una disminución de los ingresos, jubilación o discriminación laboral, entre otras condiciones $(7,8)$. Esto se encuentra íntimamente relacionado con la pobreza, en un país donde existe desigualdad en la distribución del ingreso y el porcentaje de personas que vive debajo de la línea de pobreza nacional es del $31 \%$, según datos del Banco Mundial para Colombia (2).

Es precisamente la calidad de vida y su relación con la salud, lo que en los últimos años se ha venido explorando en las diferentes poblaciones (9). Para medir esta dimensión con rigor científico, se han desarrollado diferentes instrumentos validados, y aunque el concepto es subjetivo, existen formas de cuantificar el nivel de calidad de vida, a través de encuestas donde se evalúan diferentes aspectos relacionados, donde cada ítem tiene un determinado valor y de acuerdo al puntaje final es que se califica si la calidad de vida es buena o no (10). Esta misma lógica se da para otros instrumentos relacionados con calidad de vida y la salud bucal $(11,12)$. La literatura internacional y nacional reporta estudios que evalúan la calidad de vida en muestras particulares de adultos mayores (12-15). No obstante, la investigación cualitativa ofrece un panorama amplio, al reconocer con propiedad la percepción sobre los diferentes fenómenos sociales y su relación con la salud, a través del análisis de los discursos de los directamente implicados (16). Este tipo de investigación es menos frecuente en comparación con la de corte cuantitativo (17).

Concretamente en la ciudad de Medellín (Antioquia), la segunda ciudad más grande de Colombia, existe una red hospitalaria pública, e.s.e Metrosalud, Empresa Social del Estado de primer y segundo nivel de atención que atiende un segmento poblacional importante de la ciudad, principalmente afiliados al régimen subsidiado y aquellos sin afiliación (18). Desde el año 2011 se inició un proyecto de investigación con el fin de identificar los factores determinantes de la calidad de vida y su impacto en la salud bucal en la población adulta mayor que es atendida en los servicios de salud en esta institución, con la utilización de diferentes metodologías. En un primer momento se identificaron los discursos de los prestadores de diferentes áreas de la salud y sociales (19). Posteriormente, se exploraron los discursos de los actores primarios: los adultos y adultas mayores. 
Teniendo en cuenta lo anterior, este estudio tuvo como objetivo analizar la percepción de la población adulta mayor sobre su calidad de vida, relacionándola con su salud general y su salud bucal.

\section{Materiales y métodos}

Se realizó un estudio cualitativo, descriptivo y comprensivo, mediante una perspectiva etnográfica. Con este enfoque se pretendía conocer las experiencias, vivencias y opiniones de los directamente involucrados en el entorno social y cultural: los adultos mayores (20).

Se incluyó en este estudio a la población adulta mayor con 65 y más años de edad que asistía a consulta médica y odontológica en Metrosalud. Para la selección de los participantes se contactó directamente a diferentes unidades y centros de salud para solicitar pacientes que voluntariamente aceptaran ser incluidos en esta investigación. Se realizaron 13 grupos focales (GF), los cuales fueron segmentados por sexo (6 GF de hombres y 7 GF de mujeres). Se finalizó la recolección cuando se constató la no aportación de datos nuevos sobre los objetivos de este estudio (por saturación de la información y de las categorías de análisis) $(20,21)$.

El trabajo de campo se realizó entre julio y noviembre del 2013 y los GF estuvieron a cargo del equipo investigador (AA, SA, FL, EE, CL). Se recibió un entrenamiento sobre técnicas de investigación cualitativa y se realizó una estandarización previa, con el fin de lograr entrevistas de calidad. Para la realización de los GF se contó con una guía de preguntas, las cuales fueron realizadas por el grupo investigador, la guía utilizada para orientar la entrevista se observa en la tabla 1. Se profundizó en los contenidos importantes para el objetivo del estudio y se permitió que a través del discurso libre aparecieran nuevas categorías de análisis. Se recopiló información sociodemográfica con datos como la edad, el sexo, la profesión y el nivel de estudios alcanzado. El trabajo de campo se completó con notas de observación y reuniones del grupo investigador, con el fin de precisar algunos aspectos encontrados en los GF. Participaron en los GF un total de 69 personas, siendo 31 hombres y 38 mujeres, entre 65 y 87 años de edad. Las características sociodemográficas de los participantes se enlistan en la tabla 2.

Tabla 1. Guía para la entrevista Realizada a los GRuPOS Focales (GF). Metrosalud 2013

\begin{tabular}{|l|}
\hline Categorías iniciales \\
\hline ¿Cómo es su situación de salud y salud bucal? \\
¿Cuáles son las razones para tener su salud y su \\
salud bucal como está actualmente? \\
¿Cómo es su calidad de vida? \\
¿Cómo es su calidad de vida en salud y en salud \\
bucal? \\
¿Cuál es su opinión sobre el acceso a los servicios \\
de salud? \\
¿Cómo es la situación general de los adultos \\
mayores en su barrio, en la ciudad, en Colombia? \\
¿Cuáles son sus expectativas en un futuro \\
próximo?
\end{tabular}

TABla 2. CARACTERIZACIÓN SOCIODEMOGRÁFICA DE LA POBLACIÓN DE ADULTOS MAYORES PARTICIPANTES EN LOS GRUPOS FOCALES (GF). Metrosalud, $2013(\mathrm{~N}=69 ; 13 \mathrm{GF})$

\begin{tabular}{|l|c|c|}
\hline Características & Hombres & Mujeres \\
\hline Edad & $69(65-81)$ & $72(65-87)$ \\
\hline Promedio (rango) & \multicolumn{2}{|l|}{} \\
\hline Tiempo de vivienda en el barrio \\
\hline Promedio (rango) & $31(1-70)$ & $28(1-55)$ \\
\hline Estrato socioeconómico \\
\hline Bajo (1-2) & 28 & 36 \\
\hline Medio (3-4) & 3 & 2 \\
\hline
\end{tabular}




\begin{tabular}{|c|c|c|}
\hline Características & Hombres & Mujeres \\
\hline \multicolumn{3}{|c|}{ Afiliación a la Seguridad Social } \\
\hline Régimen subsidiado & 26 & 35 \\
\hline Régimen contributivo & 5 & 3 \\
\hline \multicolumn{3}{|l|}{ Estado civil } \\
\hline Soltero & 7 & 7 \\
\hline Casado - unión libre & 22 & 20 \\
\hline Viudo & 2 & 10 \\
\hline Separado & 0 & 1 \\
\hline \multicolumn{3}{|l|}{ Nivel educativo } \\
\hline Sin estudios & 5 & 9 \\
\hline Primaria & 24 & 25 \\
\hline Secundaria & 1 & 4 \\
\hline Técnica - tecnológica & 1 & 0 \\
\hline \multicolumn{3}{|l|}{ Ocupación } \\
\hline No manual & 0 & 0 \\
\hline Manual & 11 & 4 \\
\hline Labores del hogar & 0 & 31 \\
\hline Otros & 20 & 3 \\
\hline Total & 31 & 38 \\
\hline
\end{tabular}

Los GF tuvieron una duración de entre 20 y 60 minutos, fueron grabadas en mp3 para su posterior transcripción literal en el procesador de texto Microsoft Word. Se realizó una revisión inicial con el fin de evaluar la calidad de las transcripciones y, tras repetidas lecturas de los discursos, se analizaron y se identificaron manualmente temas emergentes en los textos. Posteriormente, se hizo un análisis narrativo de contenido $(20,21)$ por medio de la identificación de fragmentos con similares significados, los textos más relevantes se presentan mediante una clave alfanúmerica que identifica el número del GF.

Este estudio cumple con los requisitos éticos para investigación en seres humanos, de acuerdo con la normativa internacional (22) y nacional (23), solicitando el consentimiento informado verbal y escrito, para participar en el estudio a los pacientes que voluntariamente colaboraron en la investigación, garantizando asimismo la confidencialidad y el respeto por la dignidad. Este estudio fue aprobado por el Comité de Ética de Investigación (CEI) de la institución Metrosalud, según acta 09-2011.

\section{Resultados}

El análisis de los resultados permitió identificar 5 categorías principales (figura 1), las cuales se exponen a continuación, separadamente para hombres y mujeres, según los discursos encontrados. Las similitudes y diferencias por sexo en las categorías analizadas se resumen en la tabla 3 .

\section{Adultas mayores (mujeres)}

\section{Salud autopercibida y factores que influyen} en el estado de salud. Las mujeres participantes en los GF manifestaron que su estado de salud general está deteriorado por la presencia de enfermedades crónicas, sin embargo, afirmaron que estas condiciones patológicas son controladas por los tratamientos médicos y terapéuticos ofrecidos dentro del Plan de Beneficios en el Sistema General de Seguridad Social en Salud.

\begin{abstract}
Mi salud hasta ahora he sido muy bien atendida por Metrosalud porque la verdad he tenido que pasar por muchas cirugías, como es del oído, las vistas y hasta ahora estoy bien, estoy bien, ahora vengo a ver cómo me acomodo a algo a ver si uno puede comer más tranquilo, porque eso también le dificulta a uno no tener con qué comer y para poder hablar. [GF 11]
\end{abstract}

Sin embargo, el panorama fue distinto cuando se les interrogó por su estado de salud bucal, pues su discurso pone en evidencia los problemas que se encuentran en su boca: caries dental, enfermedad periodontal y edentulismo parcial o total; los dos últimos 
Santiago Alzate-Urrea • Andrés A. Agudelo-Suárez • Felipe López-Vergel • Carolina LópezOrozco • Érica Espinosa-Herrera • Adriana Posada-López • Edwin J. Meneses-Gómez

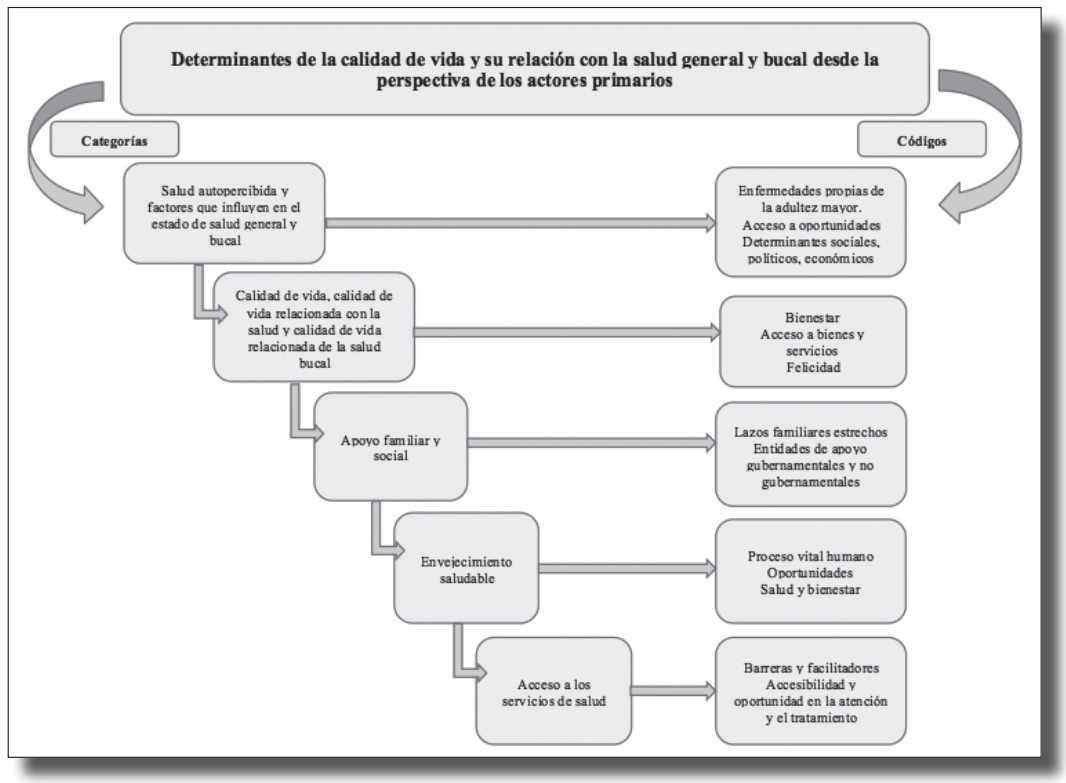

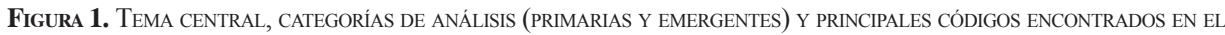
ANÁLisis de Los GRUPos Focales (GF). Metrosalud, 2013 ( $\mathrm{N}=69 ; 13 \mathrm{GF})$

no son cubiertos en su totalidad dentro del Plan de Beneficios en Salud (antes llamado Plan Obligatorio de Salud [POS]), y por tanto, no son resueltos. Además, las citas odontológicas son difíciles de conseguir si no se está en un programa especial (atención a pacientes con diabetes, hipertensión arterial).

A mí los dientes se me cayeron hace mucho rato, A mí no me falta sino que se me caiga la lengua. [GF 13]

Yo también quisiera pedir la cita para que me organizaran los dientes que no me queden así como los siento. La prótesis que me pusieron las siento como si los tuviera entaconados y las muelitas me duelen al comer. [GF 4]

Al preguntarles a las adultas mayores cuáles eran los factores que afectaron o afectan su estado de salud, ellas respondieron que el modo de vida durante su juventud y adultez temprana, las oportunidades y su desarrollo humano fueron factores protectores de su salud. Sin embargo, las oportunidades de crecimiento personal y laboral, y el acceso a bienes y servicios durante su adultez mayor son mínimos, lo cual deteriora su estado de salud, al no poder adquirir el sustento económico para poder invertir en aspectos básicos para la vida del ser humano (alimentación, recreación, salud), conjunto con el quebranto propio de la edad.

Muchas veces la alimentación, porque realmente, si la persona es demasiado pobre se come lo que pueda no más y si de pronto no tiene la leche, no tienen huevos, no tiene carne, ni pescado, ni pollo, nada, la persona se va debilitando mucho. [GF 11]

La edad, la edad que no viene sola [...] la vejez no viene sola, la vejez viene con enfermedades. [GF 6]

2. Calidad de vida ( $\mathrm{Cv}$ ), calidad de vida relacionada con la salud (CVRs) y calidad de vida relacionada con la salud bucal (CVRSB). Para las adultas mayores, el concepto de CV está ligado al acceso a bienes y servicios 
Tabla 3. Convergencias y divergencias en las Categorías analizadas entre los hombres y mujeres participantes en los Grupos focales (GF). Metrosalud, $2013(\mathrm{~N}=69 ; 13 \mathrm{GF})$

\begin{tabular}{|c|c|c|c|}
\hline \multirow{2}{*}{$\begin{array}{l}\text { Categoría de } \\
\text { análisis }\end{array}$} & \multicolumn{2}{|l|}{ Diferencias } & \multirow[t]{2}{*}{ Similitudes } \\
\hline & Hombres & Mujeres & \\
\hline $\begin{array}{l}\text { 1. Salud } \\
\text { autopercibida } \\
\text { y factores que } \\
\text { influyen en el } \\
\text { estado de salud }\end{array}$ & $\begin{array}{l}\text { Heterogeneidad en } \\
\text { el estado de salud } \\
\text { autopercibida, poca } \\
\text { o ninguna preocu- } \\
\text { pación por su salud } \\
\text { bucal }\end{array}$ & $\begin{array}{l}\text { Su salud autopercibida } \\
\text { es buena a pesar de las } \\
\text { enfermedades propias } \\
\text { de la vejez. No están } \\
\text { conformes con su } \\
\text { salud bucal }\end{array}$ & $\begin{array}{l}\text { Su modo y estilo de vida, el } \\
\text { acceso a oportunidades, bienes y } \\
\text { servicios, y el apoyo social y gu- } \\
\text { bernamental son los aspectos que } \\
\text { influyen en su estado de salud }\end{array}$ \\
\hline $\begin{array}{l}\text { 2. Calidad } \\
\text { de vida (cv), } \\
\text { calidad de vida } \\
\text { relacionada con } \\
\text { la salud (CVRs) } \\
\text { y calidad de } \\
\text { vida relaciona- } \\
\text { da con la salud } \\
\text { bucal (CVRSB) }\end{array}$ & & & $\begin{array}{l}\text { CV es el gozo máximo de las } \\
\text { capacidades y potencialidades del } \\
\text { ser humano, CVRS es tener acceso } \\
\text { adecuadamente a los servicios de } \\
\text { salud, y CVRSB se relaciona con la } \\
\text { posibilidad de tener unos dientes } \\
\text { y demás estructuras del sistema } \\
\text { estomatognático sanos estética y } \\
\text { funcionalmente }\end{array}$ \\
\hline $\begin{array}{l}\text { 3. Apoyo fami- } \\
\text { liar y social }\end{array}$ & $\begin{array}{l}\text { No reciben apoyo } \\
\text { de sus familiares } \\
\text { cercanos, pero no } \\
\text { expresan sentirse to- } \\
\text { talmente en soledad. }\end{array}$ & $\begin{array}{l}\text { Reciben el apoyo de } \\
\text { sus familiares cerca- } \\
\text { nos, por el mismo rol } \\
\text { que tienen dentro de } \\
\text { la estructura del hogar }\end{array}$ & $\begin{array}{l}\text { Reciben apoyo económico } \\
\text { subsidiado por el gobierno local y } \\
\text { departamental }\end{array}$ \\
\hline $\begin{array}{l}\text { 4. Envejeci- } \\
\text { miento salu- } \\
\text { dable }\end{array}$ & & $\begin{array}{l}\text { Para envejecer saluda- } \\
\text { blemente se requiere } \\
\text { el apoyo y la compañía } \\
\text { de los seres queridos }\end{array}$ & $\begin{array}{l}\text { Relacionado con la ausencia o } \\
\text { el control de enfermedades base } \\
\text { propias de la adultez mayor }\end{array}$ \\
\hline $\begin{array}{l}\text { 5. Acceso a los } \\
\text { servicios de } \\
\text { salud }\end{array}$ & & & $\begin{array}{l}\text { Conformidad con el acceso a la } \\
\text { consulta programada, y con el tra- } \\
\text { tamiento y la terapéutica recibida } \\
\text { Mayor acceso y oportunidad en la } \\
\text { asignación de citas médicas con } \\
\text { relación a las odontológicas }\end{array}$ \\
\hline
\end{tabular}

(alimentación adecuada, un trabajo digno para su edad y recreación en espacios donde puedan disfrutar con sus familias). La CVRS la definen como el acceso a los servicios de salud de forma adecuada, esto es, facilidad y oportunidad para la asignación y ejecución de citas, puntualidad en estas citas, respeto y responsabilidad por el adulto mayor, además de la asignación de los tratamientos y entrega de los medicamentos indicados para cada paciente. Al conversar sobre CVRSB, las mujeres asociaron esta categoría con tener los dientes en un estado adecuado tanto funcional como estéticamente.
Calidad de vida y salud, sentirse bien, desde que está enfermo, por mucha plata que tenga, no puede comer nada porque está enfermo, no puede comprarse lo que quiere porque no puede moverse, o tiene que esconderse porque lo van a secuestrar, entonces qué se gana, nada, es mejor uno ser pobre y tomarse su aguapanelita y su sopita y ya, tener tranquilidad, y ya, uno ya no está para tener plata y tener que correr, es mejor está tranquila. [GF 1]

La calidad de vida en salud bucal es en cuanto a la boca pues la dentadura obviamente, tener con qué comer, poder masticar, y para uno verse [...] la estima, autoestima. [GF 11] 
Santiago Alzate-Urrea • Andrés A. Agudelo-Suárez • Felipe López-Vergel • Carolina LópezOrozco • Érica Espinosa-Herrera • Adriana Posada-López • Edwin J. Meneses-Gómez

3. Apoyo familiar y social. Las adultas mayores se sienten apoyadas por su núcleo familiar, con algunas excepciones de mujeres que residen con familiares lejanos o vecinos, que de igual forma brindan un apoyo importante, tanto económico como psicológico, a estas mujeres. Respecto al apoyo social, se sienten respaldadas por las actividades recreativas, lúdicas y deportivas que realizan las entidades públicas, y de igual manera, el ingreso económico que reciben gracias a un programa gubernamental; este aunque es bajo, es un apoyo importante para su sustento personal.

A mí me la quitaron también y el apoyo de la familia antes yo tengo es familia que me hace harto mal a mí y es que eso es muy fuerte. [GF 13]

También el gobierno nos está dando un apoyo, un subsidio. Un subsidio que nos da es una ayudita, es una ayudita, no nos dará para todo, lógico que no, pero sí es una ayuda. [GF 13]

\section{Envejecimiento saludable. Se interrogó} a las mujeres participantes en los grupos focales sobre su concepción de un "envejecimiento saludable", término que asociaron con el hecho de llegar a la adultez mayor sin ninguna condición patológica importante, o con esta enfermedad controlada. En menor proporción, se encontró que un envejecimiento saludable debe estar precedido de factores como el acompañamiento familiar y social, y la disponibilidad de ingresos económicos suficientes para solventar las necesidades básicas, y tranquilidad emocional.

Bregar uno a comer sus cositas, arroz, verduras, ensaladitas y todo eso, pero eso uno no se lo puede comer porque no tiene cómo, para decirle la verdad, no tiene cómo. [GF 6]
Es como volver uno a la niñez, porque ya a uno se le olvidan las cosas, es distinto. [GF 4]

5. Acceso a los servicios de salud. Las adultas mayores manifestaron que el acceso a los servicios de salud, a pesar de ser aceptable en el momento de solicitar una cita por medicina general, tiene dificultades a la hora de iniciar tratamientos que no competan a los programas especiales de enfermedades de alto costo (hipertensión y diabetes). Por otra parte, los servicios odontológicos están limitados a dichos programas especiales y al servicio de atención prioritaria. Sin embargo, se sienten satisfechas con el servicio de salud que les presta la institución.

Aquí en este centro de salud que es donde nosotros venimos hace mucho tiempo, a mí me ha parecido buena la atención, lo que nos molestaba fue el tiempo que nos pusieron a pedir la cita por teléfono, que nos daba mucha lidia pedir la cita, pero ahora que ya la volvieron a integrar otra vez para pedir ficho ya es más fácil. [GF 4].

No, a mí nunca me han dado cita de odontología, cuando me duele una muela vengo. Pero si tengo dolor me atienden, sino, no. [GF 6]

\section{Adultos mayores (hombres)}

\section{Salud autopercibida y factores que in-} fluyen en el estado de salud. Los adultos mayores perciben su salud de manera heterogénea; por un lado, algunos manifiestan estar muy bien, a pesar de tener presentar enfermedades de base, controladas actualmente. Otros, por el contrario, expresan sentirse muy enfermos por patologías crónicas que padecen, y a pesar de estar en tratamiento, no sienten mejoría en su estado de salud.

Yo de salud si estoy mal, bien sea que estoy ya por manos de la internista, por ahí me están programando una ecografía de 
próstata, riñón y entonces estoy esperando a ver si me dan una mala noticia con la cita de la ecografía para llevarle los exámenes al internista. [GF 2]

Bien, me he sentido bien [...] Cómo le digo yo, no he tenido necesidad de venir, me he sentido bien, no he venido al chequeo. [GF 9]

Al preguntar por la salud bucal a los adultos mayores, manifestaron que se encontraban bien, puesto que no tenían ninguna dolencia, encontrándose que no tenían o tenían pocas piezas dentales, enfermedad periodontal y caries dental. El discurso de los participantes permitió dilucidar la poca importancia que dan al cuidado de su cavidad bucal.

Sí, para los que tienen dientes sí, para los que no tenemos dientes, no, no tenemos que preocuparnos por nada. [GF 12]

Pues haber, yo digamos con la dentadura, de la salud oral, pues al momento estoy bien [...] me hicieron un tratamiento, una prótesis que tenía pero la tenía muy deteriorada y a la vez me hicieron una revisión, unas calzas y así por el estilo. [GF 3]

Cuando se preguntó por los factores que influyeron o influían en su estado de salud, los hombres participantes en el estudio expresaron que el deterioro del ambiente en el que se vive es un factor que repercute en su salud, al igual que el autocuidado y la alimentación adecuada.

Para mí el más problema que veo yo es el medio ambiente, la contaminación, el aseo de la persona pues claro que tiene que ver con la salud, pero la contaminación está una cosa mortal. [GF 2]

2. Calidad de vida ( $\mathrm{Cv}$ ), calidad de vida relacionada con la salud (cVRs) y calidad de vida relacionada con la salud bucal (CVRSB).
Cuando se abordó el tema de Cv, los adultos mayores relacionaron este concepto con tener un buen estado de salud y un espacio adecuado donde poder desarrollar el potencial humano.

Calidad de vida, que tenga uno buena salud, lo más importante la salud que uno tenga y hasta aquí pues yo he tenido [...] a pesar de mi enfermedad he tenido buena salud. [GF 9]

La calidad de vida es que uno está libre de contaminación en primer lugar y vuelvo e insisto en eso, porque ahí tiene que ver la vista, tiene que ver con la respiración, esta cantidad de gente que hay ahora, eso es un factor. [GF 10]

La CVRS fue orientada por los hombres participantes hacia la prestación oportuna, eficaz, eficiente e integral de los servicios de salud y la aplicación de la correcta terapéutica en las patologías de cada individuo.

La calidad de vida era el tratamiento y la salud, no tiene calidad de vida, de salud, me tratan por un lado y por el otro lado me están dejando, y están dándole paso a otra. [GF 2]

La CVRSB es para los adultos mayores, la utilización de los elementos y la técnica adecuada para el cuidado de su salud bucal.

Cepillarse uno, tener una buena dentadura, usar la seda todos los días. [GF 2]

3. Apoyo familiar y social. Los adultos mayores expresaron estar laborando en empleos informales y estar viviendo con familiares lejanos o con vecinos que les rentan una habitación, y a pesar de no recibir apoyo de su familia, no expresan sentirse totalmente en soledad. Manifestaron que el acompañamiento de la institución prestadora de servicios de salud en su tratamiento es el adecuado, pues las conferencias informa- 
tivas que brindan son suficientes. Conocen los grupos de apoyo existentes en la ciudad (gimnasia pasiva, caminatas, musicoterapia), sin embargo, manifestaron que no logran conseguir un cupo en estos. En cuanto a los apoyos gubernamentales, se sienten satisfechos con el subsidio económico que les otorga la alcaldía municipal.

Yo no he podido conseguir un grupito de esos, camino mucho si, yo salgo casi todos los días a caminar una o dos horas. [GF 9]

La verdad yo no he participado, pero sí veo que hay que estar viniendo a estas charlas, nosotros tenemos las charlas para la hipertensión y yo he venido, como te dije hay veces que se me olvida y cuando vengo ya es tarde. [GF 10]

Del gobierno sí. Yo soy por adulto mayor y otros por discapacidad, yo soy discapacitado pero yo pasé la edad, siempre me dejan por adulto mayor. [GF 5]

4. Envejecimiento saludable. El proceso de envejecer sanamente fue relacionado con tener un buen estado de salud, sin enfermedades de base propias de la tercera edad.

\section{Pues envejecimiento saludable es tener buena salud. [GF 9]}

Que uno esté bien apoyado en todo [GF 5]

\section{Acceso a los servicios de salud. Para los} adultos mayores, el acceso a los servicios de salud, tanto médicos como odontológicos, es bueno; solo presentan dificultad a la hora de pedir consulta vía telefónica, porque es difícil lograr la comunicación, o por medio de turnos, porque se agotan rápidamente, y se necesita levantarse muy temprano en la mañana para ir a la unidad de salud a hacer fila, sin la seguridad de recibir un turno o la programación de una cita. Se sienten satis- fechos con los tratamientos y medicamentos ofrecidos por su institución prestadora de servicio de salud.

Pues cuando haya oportunidad que se la dan a una, muchas veces se acabaron los fichos, no es ni tan fácil porque de pronto le toca a uno madrugarse para Buenos Aires, una oficina que hay, toca hacer una fila desde las cuatro de la mañana, uno bien viejo esperando hasta las 3, 4 de la mañana que repartan fichos a las 7 de la mañana, de pronto está uno de buenas y viene uno aquí, cada mes, cada dos meses. [GF 2]

Hay días que sí se mantienen ocupados, será, pide uno una cita y que no la hay, es lo único que yo veo ahí, uno pide la cita y que para tal día [...] Sí, la cita llega. [GF 5]

Para las citas sí es como complicado pero para los medicamentos no. [GF 9]

\section{Discusión}

Los hallazgos principales de este estudio dan cuenta de una población adulta mayor que reconoce en sus discursos cómo las oportunidades y el acceso a los servicios sociales y de salud presentan algunas dificultades, esto sumado a los determinantes culturales, sociales y ambientales que se constituyen como elementos clave que inciden en la calidad de vida de esta población. Esto contrasta con la percepción de conformidad con su vida actual, en ocasiones denotando un sentimiento de resignación ante las condiciones de vulnerabilidad en las que se encuentra esta población. Por otro lado, los participantes del estudio consideran su estado de salud como bueno, argumentan en este sentido que las enfermedades crónicas que padecen son propias de la etapa del ciclo vital humano en la que se encuentran, y así normalizan su malestar físico y mental. 
La situación de salud expresada en los discursos de la población entrevistada y sus determinantes guarda relación con la literatura existente en el tema. Por ejemplo, una revisión de tema realizada por Galban $e t$ al. (24), encuentra que los adultos mayores perciben mala salud. En esa misma revisión se plantea la situación de fragilidad de esta población, teniendo en cuenta criterios médicos y funcionales, los cuales coinciden con los discursos expresados por los participantes del estudio (padecer enfermedades crónicas e invalidantes, presencia de síndromes geriátricos, malnutrición, tener una dieta no saludable, percepción de la propia actividad nula o moderada, baja actividad física, dependencia o necesidad).

En cuanto a las condiciones de salud bucal, la población entrevistada coincide en que no son las más adecuadas y esto impacta en la calidad de vida en sus dimensiones físicas y psicológicas. Un estudio en adultos mayores demandantes de atención dental en México (25) mostró que el 87,3\% de los adultos mayores presentes en su muestra tenían malestar psicológico con su boca (preocupación por el estado de salud por sus dientes y demás estructuras estomatológicas), esto concuerda con los hallazgos del presente estudio en el hecho de que las mujeres manifestaron sentimientos de incorformidad e insatisfacción con el estado de su boca, debido a diferentes causas biológicas y socioculturales, lo cual les impide desarrollarse de la mejor manera en su entorno social. En el estudio mexicano, sin embargo, la población adulta mayor cree presentar poca o ninguna dificultad en la funcionalidad biológica y social de su sistema estomatognático, a pesar de la preocupación autopercibida por su salud bucal.

En cuanto a las percepciones y las subjetividades de la población adulta mayor con relación a la calidad de vida, un estudio cualitativo desde la teoría de representacio- nes sociales llevado a cabo en Perú presenta en sus hallazgos que la calidad de vida para los adultos mayores se define como "tener paz, tranquilidad en su vida cotidiana; es ser cuidado y protegido por la familia con dignidad, afecto, respeto por su persona y por sus bienes materiales, ayudándole a lograr sus metas, permitiéndole ejercer su derecho de libre expresión, comunicación, de decisión en todo lo que se le relacione a él” (26). Dichos hallazgos están relacionados con los encontrados en Medellín, donde los adultos y las adultas mayores representaron, a través de su discurso, que la calidad de vida es tener las necesidades básicas vitales resueltas y la posibilidad de seguir desarrollándose como seres humanos. La calidad de vida es por tanto un concepto multidimensional, asociado a factores individuales y al contexto socioeconómico en el cual se insertan y desenvuelven las personas.

Con respecto a la calidad de vida relacionada con la salud (CVRS) y la calidad de vida relacionada con la salud bucal (CVRSB), es importante mencionar que hasta donde alcanza nuestro conocimiento, son escasos los estudios que establezcan una definición de estos dos conceptos desde las percepciones y representaciones de las personas. No obstante, el grupo de trabajo de la Organización Mundial de la Salud, whOQOL (27), considera que las medidas subjetivas son importantes para evaluar este constructo. Otros autores, como por ejemplo la profesora Schwartzmann (9), considera que la CVRs es determinada por factores psicológicos, orgánicos, socio-familiares, sucesos vitales, estresantes y soporte social, lo que se manifiesta en el bienestar físico, emocional y social durante el ciclo vital humano (desde el nacimiento hasta la ancianidad). La misma autora reconoce que la esencia de este concepto está en reconocer que la percepción de las personas sobre su estado de bienestar físico, psíquico, social y espiritual depende en gran parte de 
sus propios valores y creencias, su contexto cultural e historia personal. De igual manera, debe entenderse desde las oportunidades para el bienestar, las políticas de salud y las propias capacidades del individuo. Para los adultos mayores que asisten a la red hospitalaria pública de Medellín, sus percepciones van en la vía de las definiciones teóricas, encontrando que su CVRS va en relación con tener derecho a un acceso adecuado a los servicios de salud y el disfrute de su cuerpo en estado de salud.

Las redes sociales de apoyo (familia, amigos, grupos gubernamentales) son de gran importancia en el impacto positivo de la calidad de vida del adulto mayor, pues es un determinante de esta, tanto para las personas participantes de esta investigación, como en otras investigaciones sobre el tema. A este respecto, Guzmán et al. (28) desarrollan un marco conceptual para entender las redes sociales de apoyo y su papel fundamental en la calidad de vida de esta población, por el impacto demográfico en la prolongación de la esperanza de vida. Otra de las categorías analizadas en la presente investigación es el envejecimiento saludable. Ballesteros (investigadora española) menciona que para tener un envejecimiento saludable, se deben tener en cuenta los aspectos demográficos, culturales y psicosociales de cada individuo, y de igual forma se debe evaluar el proceso de envejecimiento desde el punto de vista biológico, neurológico y médico-sanitario (29). Si bien la población adulta mayor está en condición de vulnerabilidad por sus condiciones biológicas y sociales, al igual que Ballesteros, los participantes expresan en sus discursos que pueden desarrollar el concepto de "envejecer saludablemente" potenciando al máximo sus capacidades como personas y teniendo acceso al mayor número de servicios y oportunidades posible

94 para desarrollarse en los diferentes campos de la vida.
La población participante en el estudio de Medellín percibe algunos problemas de acceso para la atención con relación a los servicios de salud bucal, no obstante, se sienten satisfechas con los servicios prestados a nivel de consulta médica y aquellos complementarios a esta. Mejía et al. (30), al evaluar la equidad en el acceso a los servicios de salud, concluye que los principales factores que inciden sobre el acceso a servicios preventivos y curativos son la educación, la edad (a medida que aumenta la edad se incrementa la probabilidad de acceso por las condiciones propias de la población adulta mayor que conducen a un incremento de la demanda de los servicios de salud con relación a los más jóvenes) y el tipo de afiliación que se tiene en el Sistema General de Seguridad Social en Salud (sGsss). Otros factores relacionados son el estado de salud auto-reportado, la ubicación geográfica y el género.

Las divergencias entre hombres y mujeres están relacionadas con las esferas del desarrollo humano y los determinantes de la calidad de vida, la salud y la salud bucal no son ajenos a este fenómeno diferencial $(31,32)$. Un estudio realizado por Mora et al. (33) evaluó la perspectiva subjetiva de la CV del adulto mayor, teniendo en cuenta las diferencias ligadas al género y a la práctica de la actividad físico-recreativa, y se encontró que la calidad de vida examinada desde cuatro aspectos (autonomía, soporte social, salud mental, actividad física recreativa) presenta diferencias por sexo. Por ejemplo, las mujeres al estar expuestas durante su vida a situaciones de sometimiento en un juego de roles establecido, son más propensas a episodios de depresión geriátrica. Los adultos mayores entrevistados permitieron evidenciar en sus discursos diferencias relacionadas con las prácticas de autocuidado y en la frecuencia de uso de servicios de salud, estando a favor de las mujeres. También intervienen factores relacionados con el apoyo familiar, el cual es 
más fuerte para las adultas mayores debido al papel de cuidadoras que tuvieron con sus hijos y nietos durante su vida (32).

Como fortalezas, es preciso destacar que la investigación cualitativa en su enfoque etnográfico y fenomenológico, como herramienta para abordar temas como las percepciones y la construcción cultural de conceptos, fortalece este tipo de estudios. Se recomienda para futuros estudios realizar entrevistas individuales a adultos mayores institucionalizados en hogares geriátricos, para profundizar en los diferentes conceptos aquí estudiados y obtener una visión más amplia del fenómeno. Sin embargo, es importante tener en cuenta en la interpretación de los resultados las limitaciones del estudio. En primer lugar, es necesario resaltar los pocos reportes existentes sobre representaciones sociales, lo cual dificulta la comparación con otros estudios, aunque la investigación cualitativa pretende resaltar las particularidades de los grupos sociales. También es importante mencionar que durante los grupos focales se observaron algunas dificultades en lo que tiene que ver con la comunicación de ciertos sentimientos y afecciones por parte de los hombres de la tercera edad. Posibles explicaciones a ello radican en el fenómeno cultural e histórico de estos adultos mayores, donde los roles de género son más acentuados.

\section{Conclusiones}

Factores como el auto-reporte de la salud individual y colectiva, la percepción del nivel de calidad de vida, el sentimiento que genera el recibir o no apoyo familiar y social, y la autoconcepción del proceso de envejecimiento deben ser tenidos en cuenta a la hora de evaluar la calidad de vida de las personas y los grupos sociales de los cuales hacen parte, ya que inciden directamente en los niveles de esta. Es importante resaltar el hecho de que cada día se estudia al individuo como una entidad compleja y dinámica, que se ve influido y se afecta, positiva o negativamente, por diferentes causas de las diversas áreas del desarrollo. Por ello, las políticas y estrategias deben considerar la calidad de vida como un elemento fundamental, desde el género como una categoría analítica transversal, y abordando los determinantes sociales como una herramienta fundamental que permita identificar y disminuir las desigualdades e inequidades en los grupos especialmente vulnerables.

\section{Agradecimientos}

Los autores agradecen a la población adulta mayor que participó en los grupos focales, cuyos aportes contribuyeron al conocimiento de una realidad social importante y poco conocida.

\section{Referencias bibliográficas}

1. Cardona Arango D, Peláez E. Envejecimiento poblacional en el siglo xxI: oportunidades, retos y preocupaciones. Salud Uninorte. 2012; 28 (2): 335-48.

2. Banco Mundial. Indicadores del Banco Mundial: Esperanza de vida al nacer [acceso: 16 de marzo del 2015]. Disponible en: http://datos.bancomundial. org/indicador/SP.DYN.LE00.IN.

3. Colombia: Profamilia. Encuesta Nacional de Demografía y Salud: ENDS- 2010 [acceso: 9 de febrero del 2014]. Disponible en: http://www.profamilia org.co/encuestas/Profamilia/Profamilia/index.php.

4. Sánchez-González D, Egea-Jiménez C. Enfoque de vulnerabilidad social para investigar las desventajas socioambientales: Su aplicación en el estudio de los adultos mayores. Pap Poblac. 2011; 17 (69): 151-85.

5. Azpiazu Garrido M, Cruz Jentoft A, Villagrasa Ferrer JR, Abanades Herranz JC, García Marín N, Valero de Bernabé FA. Factores asociados a mal estado de salud percibido o a mala calidad de vida en personas mayores de 65 años. Rev Esp Salud Pública. 2002; 76 (6): 683-99.

6. Aranibar P. Acercamiento conceptual a la situación del adulto mayor en América Latina. Santiago de Chile: Centro Latinoamericano y Caribeño de Demografía (Celade) - División de Población de la Cepal. Área de Población y Desarrollo del Celade; 2001.

7. Pizarro Hofer R. La vulnerabilidad social y sus desafíos: una mirada desde América Latina. Santiago 
de Chile: División de Estadística y Proyecciones Económicas. Cepal - Naciones Unidas; 2001.

8. Salgado-de Snyder VN, Wong R. Género y pobreza: determinantes de la salud en la vejez. Salud Pública Méx. 2007; 49 (Suppl 4): s515-s21.

9. Schwartzmann L. Calidad de vida relacionada con la salud: aspectos conceptuales. Cienc Enferm. 2003; 9 (2): 9-21.

10. Misrachi C, Espinoza I. Utilidad de las mediciones de la calidad de vida relacionada con la salud. Rev Dental de Chile. 2005; 96 (2): 28-36.

11. Allen PF. Assessment of oral health related quality of life. Health Qual Life Outcomes. 2003; 1: 40.

12. Locker D, Matear D, Stephens M, Lawrence H, Payne B. Comparison of the GOHAI and OHIP-14 as measures of the oral health-related quality of life of the elderly. Community Dent Oral Epidemiol. 2001; 29 (5):373-81.

13. Duque VE, Tamayo Castrillón J, Echeverri Cadavid PA, Gutiérrez Osorio AY, Sepúlveda Correa D, Giraldo Ramírez O, et al. Calidad de vida relacionada con la salud bucal en adultos mayores que consultan a la IPS Universitaria de Medellín y sus factores asociados [Oral health related quality of life in older adults assisting "IPS Universitaria" of Medellin and associated factors]. Rev CES Odont. 2013; 26 (1): 10-23.

14. Díaz Cárdenas S, Arrieta Vergara K, Ramos Martínez K. Impacto de la salud oral en la calidad de vida de adultos mayores. Rev Clin Med Fam. 2012; 5 (1): 9-16.

15. Dahl K, Wang N, Holst D, Öhrn K. Oral healthrelated quality of life among adults 68-77 years old in Nord-Trøndelag, Norway. Int J Dent Hygiene. 2011; 9 (1): 87-92.

16. Bower E, Scambler S. The contributions of qualitative research towards dental public health practice. Community Dent Oral Epidemiol. 2007; 35 (3): 161-9.

17. Haikal DS, Paula AM, Martins AM, Moreira AN, Ferreira EF. Autopercepção da saúde bucal e impacto na qualidade de vida do idoso: uma abordagem quanti-qualitativa [Self-perception of oral health and impact on quality of life among the elderly: a quantitative-qualitative approach]. Cien Saude Colet. 2011; 16 (7): 3317-29.

18. Empresa Social del Estado (E.S.E) Metrosalud. Página web con la descripción de la E.S.E Metrosalud [acceso: 25 de febrero del 2014]. Disponible en: http://www.metrosalud.gov.co/inter/joomla/.

19. Alzate-Urrea S, López-Vergel F, López-Orozco C, Espinosa-Herrera E, Agudelo-Suárez AA, PosadaLópez A, et al. Calidad de vida y su relación con la salud bucal en la población adulta mayor atendida en la red pública hospitalaria de Medellín: la perspectiva del personal de salud. Rev CES Odont. 2014; 27 (2): 11-25.

20. Vázquez M, Ferreira Da Silva M, Mogollón A, Fernández de Sanmamed M, Delgado M IV. Intro- ducción a las técnicas cualitativas de investigación aplicadas a la salud. Santiago de Cali: Programa Editorial Universidad del Valle; 2011.

21. Patton MQ. Qualitative evaluation methods. United States of America: Sage Publications; 1980.

22. World Medical Association General A. World Medical Association Declaration of Helsinki: ethical principles for medical research involving human subjects. J Int Bioethique. 2004; 15 (1): 124-9.

23. Colombia. Ministerio de Salud. Resolución 008430 de 1993 (4 de octubre de 1993). Por la cual se establecen las normas científicas, técnicas y administrativas para la investigación en salud. Bogotá: Ministerio de Salud; 1993.

24. Alonso Galbán P, Sansó Soberats FJ, Díaz-Canel Navarro AM, Carrasco García M, Oliva T. Envejecimiento poblacional y fragilidad en el adulto mayor. Rev Cubana Salud Pública 2007; 33 (1).

25. De la Fuente Hernández J, Moreno ÓS, Valenzuela MCS, Aguilar AZ. Impacto de la salud bucal en la calidad de vida de adultos mayores demandantes de atención dental. Univ Odontol. 2010; 29 (63): 83-92.

26. Vera M. Significado de la calidad de vida del adulto mayor para sí mismo y para su familia. An Fac Med. 2007; 68 (3): 284-90.

27. The whoQol Group. The World Health Organization Quality of Life assessment (wHOQOL): position paper from the World Health Organization. Soc Sci Med. 1995; 41 (10): 1403-9.

28. Guzmán JM, Huenchuan S, Montes de Oca V. Redes de apoyo social de las personas mayores: marco conceptual. Notas de población Comisión Económica para América Latina y el Caribe (Cepal). 2003; 77: 35-70.

29. Ballesteros S. Envejecimiento saludable: aspectos biológicos, psicológicos y sociales. Madrid: Universitas. Universidad Nacional de Educación a Distancia; 2007.

30. Mejía-Mejía A, Sánchez-Gandur AF, TamayoRamírez JC. Equidad en el acceso a servicios de salud en Antioquia, Colombia. Rev Salud Pública (Bogotá). 2007; 9 (1): 26-38.

31. Kunkel SR, Atchley RC. Why gender matters: being female is not the same as not being male. Am J Prev Med. 1996; 12 (5): 294-6.

32. García Calvente MdM, Jiménez Rodrigo ML, Martìnez Morante E. Guía para incorporar la perspectiva de género a la investigación en salud. Granada: Escuela Andaluza de Salud Pública; 2010.

33. Mora M, Araya G, Ozols A. Perspectiva subjetiva de la calidad de vida del adulto mayor, diferencias ligadas al género ya la práctica de la actividad físico recreativa. MHSALUD: Revista en Ciencias del Movimiento Humano y Salud. 2004; 1 (1): 1-12. 